\title{
Thelephoraceae, Hydnaceae und Polyporaceae von Japan.
}

\author{
(Vorläufige Mitteilung.)
}

Von

Atsushi Yasuda, Rigakushi.

Dozent der Botanik an der Tōhoku Kaiserlichen Universität zu Sendai;

Professor der Zweiten Hochschule.

\section{Fam. 1. THELEPHORACEAE.}

Gen. 1. Corticium.

Sect. 1. Himantia.

Corticium laeve Pers.

Nom. Jap. Ebi-kōyaku-take.

Hab. Prov. Iyo.

Sect. 2. Lomatia.

Corticium evolvens Fr.

Nom. Jap. Shiro-köyaku-take.

Hab. Prov. Hōki, Harima.

Gen. 2. Aleurodiscus.

Aleurodiscus amorphus (Pers.) Rabenh.

Nom. Jap. Aka-kōyaku-take.

Hab. Sendai.

Aleurodiscus Oakesii (B. et C.) Cooke.

Nom. Jap. Sakazuki-koyaku-take.

Hab. Prov. Awaji. 
Gen. 3. Stereum.

Sect. 1. Resupinata.

A. Hymenium ohne Zystiden.

Stereum odoratum Fr.

Nom. Jap. Nioi-uroko-take.

Hab. Prov. Awaji, Bonin.

Stereum induratum BERK.

Nom. Jap. Miyama-uroko-take."

Hab. Berg Akagi.

Stereum medicum Curry.

Nom. Jap. Kusuri-uroko-take.

Hab. Prov. Iyo, Harima.

B. Hymenium mit Zystiden besetzt.

Stereum Mougeotii (Fr.) Cooke.

Nom. Jap. Aka-uroko-take.

Hab. Prov. Harima.

Sect. 2. Apus.

A. Hymenium ohne Zystiden.

Stereum frustulosum (Pers.) Fr.

Nom. Jap. Kata-uroko-take.

Hab. Sendai, Prov. Harima, Inaba, Awaji, Iyo.

Stereum bicolor (Pers.) Fr.

Nom. Jap. Urajiro-uroko-take.

Hab. Sendai, Prov. Rikuchū, Iwaki, Kōzuke, Mikawa, Mino, Awaji, Iyo.

Stereum fasciatum Schw.

Nom. Jap. Cha-uroko-take.

Hab. Sendai, Prov. Iwaki, Kōzuke, Iyo.

Stereum hirsutum (WILld.) Pers.

Nom. Jap. Ki-uroko-take.

Hab. Berg Akagi, Prov. Mikawa, Harima, Awaji, Iyo.

Stereum complicatum Fr.

Nom. Jap. Miyama-cha-uroko-take.

Hab. Berg Akagi, Prov. Iyo.

Stereum spadiceum (PERs.) Fr.

Nom. Jap. Chi-uroko-take.

Hab. Sendai, Prov. Rikuchū, Iwaki, Közuke, Mikawa, Mino, Harima, Iyo. 
Stereum sulcatum BuRT.

Nom. Jap. Kasa-uroko-take.

Hab. Matsuyama, Prov. Közuke.

Stereum princeps JungH.

Nom. Jap. $\bar{O}$-uroko-take.

Hab. Prov. Tosa, Mino, Harima.

Stereum membranaceum FR.

Nom. Jap. Kami-urokomtake.

Hab. Sendai, Prov. Iwaki, Közuke, Mino, Awaji, Iyo.

Stereum vibrans $B$. et $C$.

Nom. Jap. Sabi-uroko-take.

Hab. Prov. Iyo, Iwaki, Rikuchū.

Stereum spectabile Kцотz.

Nom. Jap. Momiji-uroko-take.

Hab. Prov. Tosa, Iyo, Harima, Mikawa.

B. Hymenium mit Zystiden besetzt.

Stereum tenuissimum BERK.

Nom. Jap. Kogane-uroko-take.

Hab. Sendai, Berg Akagi, Prov. Iyo.

Stereum attenuatum Lév.

Nom. Jap. Hime-uroko-take.

Hab. Berg Akagi.

Stereum tabacinum (Sow.) Fr.

Nom. Jap. Tabako-take.

Hab. Sendai, Prov. Rikuchū.

Stereum rubiginosum (Dicks.) Fr.

Nom. Jap. Ebi-uroko-take.

Hab. Sendai, Prov. Iwaki, Inaba, Iyo.

Sect. 3. Mesopus.

Hymenium ohne Zystiden.

Stereum albidum LLOyd. sp. nov.

Nom. Jap. Sagi-uroko-take.

Hab. Prov. Kōzuke.

Stereum Burtianum PECK.

Nom. Jap. Hana-uroko-take.

Hab. Sendai, Prov. Iwaki, Kōzuke, Harima, Inaba. 


\section{Stereum Harmandi PAT.}

Nom. Jap. Kiburi-uroko-take.

Hab. Sendai.

Stereum elegans MEyer.

Nom. Jap. Tachi-uroko-take.

Hab. Prov. Mikawa.

Gen. 4. Thelephora.

Sect. 1. Resupinatae.

Thelephora penicillata (Pers.) Fr.

Nom. Jap. Sasara-take.

Hab. Sendai, Prov. Iwaki.

Sect. 2. Pileatae.

Thelephora laciniata Pers.

Nom. Jap. Nokogiri-uroko-take.

Hab. Prov. Rikuzen.

Sect. 3. Erectae.

Thelephora palmata (Scop.) Fr.

Nom. Jap. Kumade-take.

Hab. Sendai, Prov. Mikawa, Inaba.

Thelephora papillosa Lioyd. sp. nov.

Nom. Jap. Tsubu-ibo-take.

Hab. Prov. Mikawa, Iyo.

Thelephora japonica Yasuda. sp. nov.

Nom. Jap. Ibo-take.

Hab. Sendai.

Gen. 5. Craterellus.

Sect. Tubaeformes.

Craterellus aureus B. et C. .

Nom. Jap. Tokiiro-rappa-take.

Hab. Sendai, Prov. Harima, Iyo.

Craterellus cornucopioides (L.) Pers.

Nom. Jap. Kuro-rappa-take.

Hab. Sendai. 


\title{
Fam. 2. HYDNACEAE.
}

\author{
Gen. 1. Phlebia.
}

Phlebia strigoso-zonata (Scrw.) LLovd.

Nom. Jap. Shiwa-uroko-take.

Hab. Prov. Iyo, Harima, Shinano, Kōzuke, Rikuchū.

\section{Gen. 2. Lopharia.}

Lopharia jayanica Hens.

Nom. Jap. Kushinoha-shiwa-take.

Hab. Prov. Mikawa, Harima, Awaji, Iyo.

\section{Gen. 3. Radulum.}

Radulum molariforme (PERs.) LLOyd.

Nom. Jap. Okuba-take.

Hab. Sendai.

\section{Gen. 4. Hydnochaete.}

Hydnochaete japonica LLOYD. sp. nov.

Nom. Jap. Samegawa-take.

Hab. Bonin.

\section{Gen. 5. Hydnum.}

Sect. 1. Apus.

Hydnum ochraceum GMEL.

Nom. Jap. Niku-hari-take.

Hab. Sendai, Berg Akagi, Prov. Rikuchū, Awaji, Iyo.

Hydnum discolor FR.

Nom. Jap. Atsumi-hari-take.

Hab. Prov. Kōzuke.

Sect. 2. Merisma.

Hydnum Erinaceus Bull.

Nom. Jap. Yamabushi-take.

Hab. Berg Akagi.

Hydnum helvolum LÉv.

Nom. Jap. Ōgi-hari-take.

Hab. Berg Akagi. 
Sect. 3. Pleuropus.

Hydnum adustum ScHw.

Nom. Jap. Chaura-hari-take.

Hab. Prov. Iwaki.

Hydnum Auriscalpium L.

Nom. Jap. Matsukasa-take.

Hab. Prov. Hōki.

Sect. 4. Mesopus.

A. Lignosa.

a. Sporen farblos.

Hydnum cyathiforme SchaEfF.

Nom. Jap. Jogo-hari-take.

Hab. Prov. Mikawa, Shinano.

Hydnum concrescens Pers.

Nom. Jap. Ō-cha-hari-take.

Hab. Sendai.

Hydnum nigrum $F$.

Nom. Jap. Shibu-hari-take.

Hab. Sendai, Prov. Kōzuke, Mikawa, Harima.

Hydnum alboniger Peck.

Nom. Jap. Shibu-hari-take-modoki.

Hab. Prov. Kōzuke.

Hydnum melaleucum $F_{R}$.

Nom. Jap. Kuri-hari-take.

Hab. Sendai, Prov. Owari.

Hydnum grayeolens (Pers.) Fr.

Nom. Jap. Kuro-hari-take.

Hab. Sendai.

b. Sporen gefärbt.

Hydnum aurantiacum (Batsch) Pers.

Nom. Jap. Ki-hari-take.

Hab. Prov. Mikawa.

Hydnum conigenum Peck.

Nom. Jap. Kaki-hari-take.

Hab. Prov. Iwashiro, Kōzuke.

Hydnum zonatum Batsch.

Nom. Jap. Cha-hari-take.

Hab. Sendai, Prov. Shinano, Mikawa, Harima, Iyo. 
B. Carnosa.

a. Sporen farblos.

Hydnum albidum Peck.

Nom. Jap. Hime-hari-take.

Hab. Sendai.

Hydnum repandum L.

Nom. Jap. Kanoshita.

Hab. Sendai.

b. Sporen gefärbt.

Hydnum scabrosum Fr.

Nom. Jap. Ke-rōji.

Hab. Prov. Mikawa.

Hydnum imbricatum L.

Nom. Jap. Kanohana.

Hab. Sendai.

Hydnum aspratum BERK.

Nom. Tap. Kö-take.

Hab. Sendai, Prov. Uzen.

Gen. 6. Irpex.

Sect, 1. Resupinati.

Irpex obliquus (Schrad.) Fr.

Nom. Jap. Nagaba-take.

Hab. Prov. Iwashiro.

Sect. 2. Sessiles.

Irpex lacteus $F_{R}$.

Nom. Jap. Usuba-take.

Hab. Sendai, Prov. Kōzuke.

Irpex consors BERK.

Nom. Jap. Koba-no-usuba-take.

Hab. Prov. Owari.

Irpex Tanakae MurRILL.

Nom. Jap. Ami-usuba-take.

Hab. Sendai, Prov. Rikuchū, Iwaki, Sagami, Kōzuke, Mikawa, Mino, Harima, Hōki, Izumo, Awaji, Iyo.

Irpex japonicus MurRILL.

Nom. Jap. Usuba-take-modoki.

Hab. Sendai, Prov. Iyo, -Awaji, -Hôki, Bōshū. 
Irpex iyoensis Yasuda. sp. nov.

Nom. Jap. Ehime-usuba-take.

Hab. Matsuyama.

\section{Fam. 3. POLYPORACEAE.}

Subfam. 1. Merulieae.

Gen. 1. Merulius.

Sect. Leptospori.

Merulius tremellosus Schrad.

Nom. Jap. Shiwa-take.

Hab. Berg. Akagi, Prov. Iyo.

Merulius castaneus Lroyd. sp. nov.

Nom. Jap. $\bar{O}$-shiwa-take.

Hab. Sendai, Nagoya, Matsuyama, Prov. Közuke.

Subfam. 2. Polyporeae.

Gen. 1. Poria.

Sect. 1. Vaporariae.

Poria yaporaria Pers.

Nom. Jap. Watagusare-take.

Hab. Prov. Ishikari.

Poria xylina Lroyd. sp. nov.

Nom. Jap. $\bar{O}$-ana-take.

Hab. Formosa.

Sect. 2. Molluscae.

Poria yersipora Pers.

Nom. Jap. Ana-take.

Hab. Prov. Iwashiro, Iyo.

Poria callosa $F_{R}$.

Nom. Jap. Kata-ana-take.

Hab. Prov. Iyò.

Poria aurantia-tingens Ellis.

Nom. Jap. Kizome-take.

Hab. Bonin. 
Gen. 2. Fomes.

Sect. 1. Pallidus.

Fomes connatus Fr.

Nom. Jap. Shiro-saru-no-koshikake.

Hab. Prov. Iwashiro.

Fomes annosus $F_{R}$.

Nom. Jap. Matsu-no-nekuchi-take.

Hab. Sendai, Prov. Iyo.

Fomes pinicola $F_{R}$.

Nom. Jap. Tsuga-saru-no-koshikake.

Hab. Sendai, Sapporo, Nikkō, Berg Kōya, Prov. Iyo.

Fomes subungulatus MurRIrI.

Eine Form von Fomes pinicola Fr.

Nom. Jap. Hime-tsuga-saru-no-koshikake.

Hab. Prov. Iyo.

Sect. 2. Fuscus.

A. Hymenium ohne Zystiden ; Sporen farblos.

Fomes odoratus (Wulf.) Lloyd.

Nom. Jap. Nioi-ami-take.

Hab. Berg Fuji.

Fomes fomentarius (L.) Fr.

Nom. Jap. Hokuchi-take.

Hab. Sendai, Sapporo, Prov. Uzen, Iwashiro, Kōzuke, Iyo.

Fomes angularis Umemura. sp. nov.

Nom. Jap. Chizugata-saru-no-koshikake.

Hab. Prov. Mikawa.

Fomes pusillus Yasuda. sp. nov.

Nom. Jap. Hime-hizume-take.

Hab. Prov. Kōzuke, Harima.

Fomes melanoporus Mont.

Nom. Jap. Kuro-saru-no-koshikake.

Hab. Sendai.

B. Hymenium mit Zystiden besetzt; Sporen farblos.

Fomes robustus KarsT.

Nom. Jap. Momi-saru-no-koshikake.

Hab. Sendai, Prov. Iyo, Mino, Kazusa. 


\section{Fomes Bakeri Murrill.}

- Eine Form von Fomes robustus Karst.

Nom. Jap. Kashi-sart-no-koshikake.

Hab. Prov. Iyo.

\section{Fomes torulosus Pers.}

Nom. Jap. Koruku-take.

Hab. Sendai, Prov. Mikawa, Mino, Iyo, Bonin.

Fomes lamaensis Murrill.

Nom. Jap. Shima-saru-no-koshikake.

Hab. Bonin.

Fomes pini (Brot.) Lloyd.

Nom. Jap. Matsu-no-katawa-take.

Hab. Prov. Iburi.

C. Hymenium ohne Zystiden; Sporen gefärbt.

\section{Fomes minutulus Henn.}

Nom. Jap. Hime-hokuchi-take.

Hab. Berg Akagi.

Fomes Caryophylli (RAc.) LLoyd.

Nom. Jap. Kurogane-take.

Hab. Berg Akagi.

D. Hymenium mit Zystiden besetzt; Sporen gefärbt.

\section{Fomes pachyphloeus Pat.}

Nom. Jap. Kurosuji-saru-no-koshikake.

Hab. Berg Fuji.

Sect. 3. Ganodermus.

Fomes applanatus (Pers.) W $\mathrm{W}_{\text {ALIR. }}$

Nom. Jap. Kofuki-saru-no-koshikake.

Hab. Sendai, Prov. Iwashiro, Mikawa, Mino, Iyo.

Fomes leucophaeus Mont.

Eine Form von Fomes applanatus (PERs.) WALLR.

Nom. Jap. Tsune-no-saru-no-koshikake.

Hab. Sendai, Prov. Ishikari, Iwashiro, Kazusa, Bōshū, Kōzuke, Mikawa, Awaji.

Fomes nigro-laccatus Cooke.

Eine Form von Fomes applanatus (Pers.) WallR.

Nom. Jap. Yani-saru-no-koshikake.

Hab. Berg Akagi, Matsuyama, Formosa. 


\section{Gen. 3. Polyporus.}

Sect. 1. Suberosi.

Polyporus sambuceus LLoyd. sp. nov.

Nom. Jap. Shiro-kaimen-take.

Hab. Sendai, Berg Akagi, Prov. Iwaki, Iwashiro, Shinano, Mikawa, Inaba, Iyo.

Polyporus betulinus (BULı.) Fr.

Nom. Jap. Kamba-take.

Hab. Prov. Kōzuke.

Polyporus resinosus (Schrad.) Fr.

Nom. Jap. Yani-take.

Hab. Prov. Rikuchū.

\section{Sect. 2. Pallidi.}

A. Durus.

Polyporus ostreiformis BERK.

Nom. Jap. Kakigara-take.

Hab. Angaur.

Polyporus foedatus Berk.

Nom. Jap. Mogusa-take.

Hab. Sendai, Bonin.

Polyporus ochroleucus Berk.

Nom. Jap. Uzura-take.

Hab. Sendai, Tōkyō, Prov. Mikawa, Iyo.

Polyporus yersisporus Yasuda. sp. nov.

Nom. Jap, $\bar{O}$-uzura-take.

Hab. Prov. Tosa, Iyo, Palau, Truk, Ponape.

Polyporus volvatus Peck.

Nom. Jap. Hitokuchi-take.

Hab. Sendai, Prov. Kōzuke, Ōsumi.

\section{B. Mollis.}

Polyporus trabeus Fr.

Nom. Jap. Oshiroi-take.

Hab. Sendai, Berg Akagi, Prov. Mikawa, Harima, Iyo.

Polyporus ravidus $F_{R}$.

Nom. Jap. Zaimoku-take.

Hab. Sendai, Prov. Harima, Inaba. 
Sect. 3. Dichroi.

Polyporus adustus (WILLd.) FR.

Nom. Jap. Yakeiro-take.

Hab. Prov. Kōzuke, Mikawa, Iyo.

Polyporus secernibilis Berk.

Eine form von Polyporus adustus (WILLd.) FR.

Nom. Jap. Hime-yakeiro-take.

Hab. Sendai, Prov. Iyo, Awaji, Inaba, Harima, Mikawa.

Polyporus amorphus Fr.

Nom. Jap. Urabeni-take.

Hab. Prov. Közuke, Harima, Iyo.

Polyporus dichrous Fr.

Nom. Jap. Ebiura-take.

Hab. Sendai, Prov. Iwaki, Iyo.

Sect. 4. Fusci.

A. Hymenium ohne Zystiden; Sporen farblos.

Polyporus zonalis BERK.

Nom. Jap. Surume-take.

Hab. Prov. Shinano, Mikawa.

Polyporus rigidus Lév.

Eine Form von Polyporus zonalis BERK.

Nom. Jap. Surume-take-modoki.

Hab. Bonin, Prov. Mikawa.

Polyporus semilaccatus BERK.

Nom. Jap. Bekkō-take.

Hab. Sendai, Prov. Iyo.

Polyporus vinosus Berk.

Nom. Jap. Budō-take.

Hab. Prov. Iyo, Harima, Mikawa, Kōzuke.

B. Hymenium mit Zystiden besetzt; Sporc $n$ farblos.

Polyporus gilvus ScHw.

Nom. Jap. Nendo-take.

Hab. Berg Akagi, Prov. Iyo.

Polyporus scruposus Fr.

Eine Form von Polyporus gilvus ScHw.

Nom. Jap. Kimen-take.

Hab. Angaur. 
Polyporus illicicola Henn.

Eine Form von Polyporus gilvus Schw.

Nom. Jap. Daidai-take.

Hab. Sendai, Prov. Rikuchū, Iwaki, Shinano, Mikawa, Mino, Harima, Iyo.

C. Hymenium ohne Zystiden; Sporen gefärbt.

Polyporus caryophylleus Cooke.

Nom. Jap. Chöji-take.

Hab. Sendai, Berg Akagi, Bonin.

Polyporus pubertatis YASUdA. sp. nov.

Nom. Jap. Hōnen-take.

Hab. Prov. Közuke.

D. Hymenium mit Zystiden besetzt; Sporen gefärbt.

Polyporus Mikadoi Umemura. sp. nov.

Eine Form von Polyportus cuticularis (BULL.) Fr.

Nom. Jap. Kawaoso-take.

Hab. Sendai, Tōkyō, Berg Akagi, Prov. Iwaki, Harima, Iyo.

Sect. 5. Ganoderma.

Polyporus lucidus (Leys.) Fr.

Nom. Jap. Shima-mannen-take.

Hab. Bonin, Saipan.

Polyporus yalesiacus Boudier.

Eine Form von Polyporus lucidus (Leys.) Fr.

Nom. Jap. $\bar{O}$-mannen-take.

Hab. Prov. Iwashiro.

Polyporus japonicus Fr.

Eine Form von Polyporus lucidus (LEYs.) Fr.

Nom. Jap. Mannen-take, Saiwai-take.

Hab. Sendai, Tökyō, Prov. Kazusa, Awaji, Iyo, Formosa.

Polyporus oregonensis MURRILL.

Nom. Jap. Mannen-take-modoki.

Hab. Prov. Kōzuke, Shinano.

Sect. 6. Lignosi.

Folyrorus musashiensis Hens:

Nom. Jap. Musashi-take.

Hab. Prov. Mikawa. 
Sect. 7. Merisma.

Polyporus umbellatus (Pers.) Fr.

Nom. Jap. Chorei.

Hab. Prov. Iwashiro.

Polyporus frondosus Fr.

Nom. Jap. Mai-take.

Hab. Sendai.

Polyporus dispansus Yasuda. sp. nov.

Nom. Jap. Kömori-take.

Hab. Sendai, Prov. Mino.

Polyporus sulphureus (BuLL.) Fr.

Nom. Jap. Aikawa-take, Masu-take.

Hab. Sendai, Prov. Kōzuke, Awaji, Iyo, Bonin.

Sect. 8. Petalloides.

Polyporus pusillus Pers.

Nom. Jap. Suzume-take.

Hab. Bonin, Prov. Iyo.

Polyporus Mikawai LLoyd. sp. nov:

Nom. Jap. Mikawa-take.

Hab. Prov. Mikawa.

Polyporus ochro-tinctus B. et C.

Nom. Jap. Bisketto-take.

Hab. Prov. Közuke.

Polyporus Yoshinagai Lloyd. sp. nov.

Nom. Jap. Yokogura-take.

Hab. Berg Yologura.

Polyporus Pocula (Schw.) B. et C.

Nom. Jap. Nurude-take.

Hab. Sendai, Prov. Rikuchū, Iyo.

Sect. 9. Pelloporus.

Polyporus Cumingii Berk.

Nom. Jap. Saji-take.

Hab. Sendai, Berg Akagi, Prov. Iyo.

Polyporus scaurus LLoyd. sp. nov.

Nom. Jap. Shakushi-take.

Hab. Sendai, Prov. Shinano, Kōzuke.

Polyporus orientalis Mumemura. sp. nov.

Nom. Jap. Azuma-take.

Hab. Prov. Mikawa, Inaba, Awaji, Iyo.. 
Sect. 10. Melanopodes.

Polyporus yarius (Pers.) Fr.

Nom. Jap. Ashiguro-take.

Hab. Sendai, Berg Akagi, Prov. Iyo.

Polyporus Guilfoylei B. et BR.

Nom. Jap. Suji-uchiwa-take.

Hab. Bonin.

\section{Sect. 11. Spongiosi.}

Polyporus heteroporus Fr.

Eine Form von Polyporus rufescens (Pers.) Fr.

Nom. Jap. Niku-uchiwa-take.

Hab. Sendai, Prov. Rikuchū, Harima, Inaba.

\section{Polyporus Schweinitzii Fr.}

Nom. Jap. Kaimen-take.

Hab. Prov. Mikawa.

\section{Sect. 12. Lenti.}

Polyporus arcularius (BATSCH) Fr.

Nom. Jap. Ami-sugi-take.

Hab. Sendai, Prov. Iwaki.

Sect. 13. Ovini.

Polyporus squamosus (Huds.) Fr.

Nom. Jap. Cha-uchiwa-take.

Hab. Prov. Mikawa.

Polyporus confluens (ALB. et Schw.) Fr.

Nom. Jap. Ningyō-take.

Hab. Sendai.

Polyporus Cantharellus LLoyd. sp. nov.

Nom. Jap. Ami-rappa-take.

Hab. Sendai.

Polyporus Yasudai Lloyd. sp. nov.

Nom. Jap. Numeri-ai-take.

Hab. Sendai.

Polyporus leucomelas (Pers.) Fr.

Nom. Jap. Kurokawa, Rōji.

Hab. Sendai, Prov. Mikawa. 
Gen. 4. Polystictus.

Sect. 1. Coriacei.

A. Versicolores.

Polystictus versicolor (L.) Fr.

Nom. Jap. Kawara-take.

Hab. Sendai, Prov. Iwashiro, Mikawa, Mino, Harima, Awaji, Iyo.

Polystictus hirsutus $\mathrm{F}_{\mathrm{R}}$.

Nom. Jap. Arage-kawara-take.

Hab. Prov. Echigo, Hōki.

Polystictus hirsutus Fr., f. albidus LLoyd.

Nom. Jap. Shiro-kawara-take.

Hab. Sendai, Prov. Iyo, Awaji, Harima, Mino, Iwashiro.

Folystictus hirsutus Fr., f. pores adustus LLovd.

Nom. Jap. Chaura-shiro-kawara-take.

Hab. Sendai, Prov. Iyo.

Polystictus hirsutulus Schw.

Nom. Jap. Ke-kawara-take.

Hab. Sendai.

Polystictus polyzonus Pers.

Nom. Jap. Kitsune-kawara-take.

Hab. Sendai, Prov, Mikawa, Awaji, Tosa.

Polystictus vellereus BERK.

Nom. Jap. Ami-kawara-take.

Hab. Prov. Mikawa, Iyo.

Polystictus pubescens (Schum.) Fr.

Eine Form von Polystictus velutinus Fr.

Nom. Jap. Yakifu-take.

Hab. Prov. Közuke.

Polystictus nigro-marginatus ScHw.

Nom. Jap. Heritori-take.

Hab. Sendai, Prov. Iyo, Awaji, Harima, Mikawa, Kōzuke, Iwaki, Rikuchū.

Polystictus abietinus Fr.

Nom. Jap. Shihai-take.

Hab. Sendai, Prov. Kōzuke. 
B. Scortei.

Polystictus Persoonii Fr.

Nom. Jap. Rengwa-take.

Hab. Sendai, Prov. Iwaki, Kazusa, Harima, Iyo, Formosa, Truk.

C. Lutescentes.

Polystictus luteo-olivaceus B. et BR.

Nom. Jap. Sembei-take.

Hab. Prov. Mikawa.

D. Caperati.

Polystictus jodinus MonT.

Nom. Jap. Kinuhada-take.

Hab. Prov. Iyo, Awaji, Mikawa, Bōshū.

Sect. 2. Stuposi.

Polystictus biformis KLOTZ.

Nom. Jap. Mino-take.

Hab. Berg Akagi, Prov. Iwaki.

Polystictus pergamenus Fr.

Nom. Jap. Ha-kawara-take.

Hab. Prov. Iwaki, Iyo.

Polystictus yersatilis BERK.

Nom. Jap. Shirage-take.

Hab. Sendai, Prov. Kōzuke, Iyo.

Sect. 3. Discipedes.

Polystictus glabratus Yasuda. sp. nov.

Nom. Jap. Shiro-ögi-take.

Hab. Sendai.

Polystictus elongatus BERK.

Nom. Jap. Shiro-ha-kawara-take.

Hab. Prov. Izumo, Inaba.

Polystictus gallo-payonis BERK. et BR.

Nom. Jap. Karakun-take.

Hab. Angaur.

Polystictus nipponicus YASUDA. sp. nov.

Nom. Jap. Akagi-take.

Hab. Berg Akagi, Prov. Rikuchū. 
Polystictus affinis NEEs.

Nom. Jap. Tsuya-uchiwa-take.

Hab. Sendai, Prov. Iwashiro, Bōshū, Kōzuke, Mikawa, Harima, Inaba, Izumo, Awaji, Awa, Iyo, Tosa.

Polystictus luteus Blum. et Nees.

Nom. Jap. Shima-uchiwa-take.

Hab. Bonin.

Polystictus flabelliformis KLOTz.

Nom. Jap. Uchiwa-take.

Hab. Bonin.

Polystictus subaffinis LLOyd. sp. nov.

Nom. Jap. Tsuya-uchiwa-take-modoki.

Hab. Tōkyō, Prov. Mikawa.

Polystictus pterygodes Fr.

Nom. Jap. Tsubasa-take.

Hab. Bonin.

Polystictus vernicipes Berk.

Nom. Jap. Wanisu-take.

Hab. Bonin.

Polystictus neaniscus BERK.

Nom. Jap. Ura-gin-take.

Hab. Prov. Iyo, Awaji, Harima, Közuke.

Polystictus sanguineus (L.) FR.

Nom. Jap. Hiiro-take.

Hab. Sendai, Prov. Iwaki, Kōzuke, Mino, Harima, Awaji, Iyo, Bonin, Formosa.

Sect. 4: Pelloporus.

Polystictus perennis (L.) FR.

Nom. Jap. Otsunen-take.

Hab. Prov. Kōzuke, Iyo.

Polystictus subpictus LLOYD. sp. nov.

Nom. Jap. Hime-nikkei-take.

Hab. Prov. Mikawa.

Polystictus cinnamomeus JACQ.

Nom. Jap. Nikkei-take.

Hab. Prov. Iyo, Inaba, Mikawa, Kōzuke, Iwaki.

Polystictus dependens B. et C.

Nom. Jap. Hime-kaimen-take.

Hab. Sendai, Prov. Harima, Iyo. 
Gen. 5. Trametes.

Sect. 1. Sessiles.

Trametes vittata (BERK.) LLOYd.

Nom. Jap. Mompa-take.

Hab. Prov. Iyo, Harima, Mino, Mikawa; Kōzuke.

Trametes Trogii BERK.

Nom. Jap. Usagi-take.

Hab. Berg Akagi.

\section{Trametes Bulliardii Fr.}

Eine Trametes-Form von Daedalea confragosa (BolT.) PERs.

Nom. Jap. Cha-ami-take.

Hab. Prov. Iwashiro, Rikuchū.

Trametes confragosa LLOYD. nov.

Eine Trametes-Form von Daedalea confragosa (BoLT.) Pers.

Nom. Jap. Miiro-ami-take.

Hab. Berg Akagi, Prov. Harima, Iyo.

Trametes Dickinsii (BERK.) Lloyd.

Nom. Jap. Höroku-take.

Hab. Sendai, Berg Akagi, Prov. Echigo, Iwashiro, Harima, Awaji, Iyo.

Trametes Mülleri BERK.

Nom. Jap. Kawarake-take.

Hab. Prov. Mikawa, Awaji, Iyo, Bonin, Formosa.

Trametes cervinus (Pers.) Lloyd.

Nom. Jap. Shika-take.

Hab. Berg Akagi, Prov. Iyo, Rikuchū.

Sect. 2. Scutatae.

Trametes heteropora MonT.

Nom. Jap. Shima-ami-take.

Hab. Formosa.

\section{Gen. 6. Daedalea.}

Sect. 1. Subcoriaceae.

Daedalea unicolor (BULL.) Fr.

Nom. Jap. Midare-ami-take.

Hab. Sendai, Prov. Echigo, Kōzuke, Iyo. 
Sect. 2. Suberoso-lignosae.

Daedalea gibbosa Pers.

Nom. Jap. Shiro-midare-ami-take.

Hab. Berg Fuji.

Daedalea Kusanoi Murrill.

Nom. Jap. $\bar{O}$-midare-ami-take.

Hab. Tōkyō, Prov. Awa.

Daedalea confragosa (Bolt.) Pers.

Nom. Jap. Cha-midare-ami-take.

Hab. Sendai, Berg Akagi.

Daedalea ungulata LLoyd. sp. nov.

Nom. Jap. Hizume-midare-ami-take.

Hab. Prov. Iyo.

\section{Gen. 7. Lenzites.}

Sect. 1. Tomentosae v. pubescentes.

\section{Lenzites Earlei MurriLL.}

Nom. Jap. Ō-chirimen-take.

Hab. Prov. Hōki.

Lenzites betulina (L.) FR.

Nom. Jap. Kaigara-take.

Hab. Sendai, Prov. Iwaki, Iwashiro, Kōzuke, Harima, Inaba, Hōki, Iyo.

Lenzites japonica $\mathrm{B}$. et $\mathrm{C}$.

Nom. Jap. $\bar{O}$-kaigara-take.

Hab. Prov. Shinano.

Lenzites sepiaria (WuLF.) Fr.

Nom. Jap. Ki-kaigara-take.

Hab. Prov. Inaba.

Lenzites subferruginea BERK.

Nom. Jap. Hiroha-no-ki-kaigara-take.

Hab. Sendai, Prov. Echigo, Iwaki, Kōzuke, Mikawa, Mino, Kii, Harima, Inaba, Hōki, Izumo, Awaji, Iyo.

Lenzites striata SwARTz.

Nom. Jap. Hime-ki-kaigara-take.

Hab. Sendai, Prov. Echigo, Iwaki, Kōzuke, Harima, Awaji, Iyo, Formosa.

Lenzites tricolor (BULL.) FR.

Nom. Jap. Cha-kaigara-take.

Hab. Sendai, Prov. Iyo, Inaba, Suruga, Iwaki, Rikuchū. 
Lenzites styracina (HenN et ShIRAI.) Lloovd.

Nom. Jap. Egonoki-take.

Hab. Prov. Rikuchū, Echigo, Kōzuke, Iyo.

Sect. 2. Glabrae v. glabrescentes.

Lenzites repanda (MonT.) Fr.

Nom. Jap. Chirimen-take.

Hab. Prov. Közuke, Inaba, Iyo.

Gen. 8. Hexagonia.

Sect. Sulcatae.

Hexagonia bixalyis (PERs.) Llooyd.

Nom. Jap. Furui-take.

Hab. Bonin, Truk.

Gen. 9. Fayolus.

Sect. Pleuropodes.

Fayolus europaeus Fr.

Nom. Jap. Hachinosu-take.

Hab. Sendai, Prov. Kōzuke, Iyo.

Gen. 10. Cyclomyces.

Cyclomyces fuscus Fr.

Nom. Jap. Wahida-take.

Hab. Prov. Iyo, Höki, Mikawa.

Cyclomyces Greenii Berk.

Nom. Jap. Uzu-take.

Hab. Prov. Közuke.

Subfam. 3. Boleteae.

Gen. 1. Boletus.

Sect. Euchroi.

Boletus bovinus L.

Nom. Jap. Ami-take.

Hab. Sendai, Prov.Mikawa, Iyo.

Gen. 2. Strobilomyces.

Strobilomyces strobilaceus (Scop.) BERK.

Nom. Jap. Oni-iguchi.

Hab...Sendai. 
Strobilomyces pallidus Cooke.

Nom. Jap. Hime-oni-iguchi.

Hab. Prov. Inaba.

Gen. 3. Boletopsis.

Boletopsis luteus (L.) Henn.

Nom. Jap. Numeri-iguchi.

Hab. Sendai, Prov. Kōzuke.

Naturwissenschaftliche Fakultät der Tōhoku Kaiserlichen Universität zu Sendai, 20. Januar 1917. 